\title{
Multifrequency OFDM SAR in Presence of Deception Jamming
}

\author{
Jonathan Schuerger ${ }^{1,2}$ and Dmitriy Garmatyuk ${ }^{1}$ \\ ${ }^{1}$ Department of Electrical and Computer Engineering, Miami University, Oxford, OH 45056, USA \\ ${ }^{2}$ Raytheon Company, El Segundo, CA 90245, USA
}

Correspondence should be addressed to Dmitriy Garmatyuk, garmatd@muohio.edu

Received 31 May 2009; Revised 18 October 2009; Accepted 12 January 2010

Academic Editor: Laurent Ferro-Famil

Copyright (C) 2010 J. Schuerger and D. Garmatyuk. This is an open access article distributed under the Creative Commons Attribution License, which permits unrestricted use, distribution, and reproduction in any medium, provided the original work is properly cited.

Orthogonal frequency division multiplexing $(\mathrm{OFDM})$ is considered in this paper from the perspective of usage in imaging radar scenarios with deception jamming. OFDM radar signals are inherently multifrequency waveforms, composed of a number of subbands which are orthogonal to each other. While being employed extensively in communications, OFDM has not found comparatively wide use in radar, and, particularly, in synthetic aperture radar (SAR) applications. In this paper, we aim to show the advantages of OFDM-coded radar signals with random subband composition when used in deception jamming scenarios. Two approaches to create a radar signal by the jammer are considered: instantaneous frequency (IF) estimator and digital-RF-memory(DRFM-) based reproducer. In both cases, the jammer aims to create a copy of a valid target image via resending the radar signal at prescribed time intervals. Jammer signals are derived and used in SAR simulations with three types of signal models: OFDM, linear frequency modulated (LFM), and frequency-hopped (FH). Presented results include simulated peak side lobe (PSL) and peak cross-correlation values for random OFDM signals, as well as simulated SAR imagery with IF and DRFM jammers'-induced false targets.

\section{Introduction}

Synthetic aperture radar (SAR) technology has been used since the 1960's for the purposes of imaging landscapes and seascapes-both for the civilian and military purposes [1]. In the latter scenarios, the enemy may frequently use specific electronic countermeasures (ECM) [2] to introduce false imagery into the radar-collected data to prevent accurate battle scene assessment. Such methods of ECM are classified as deceptive [3]. Deceptive ECM techniques_-or deception jammers - operate by sensing incoming radar signals and reproducing them to the best of jammer's capabilities, then resending resultant pulses in a particular fashion, so as to hinder correct imaging of enemy targets. False Target Generation (FTG) is one such commonly used form of deception jamming. The replicated and delayed SAR waveform is transmitted at the next expected arrival of the radar signal and is seen as an actual target after image reconstruction. This type of FTG can be accomplished using a digital radio frequency memory (DRFM) repeat jammer [4].
Another possible approach to FTG is to generate the replica waveforms by determining the instantaneous frequency (IF) of the incoming radar signal [5]. The effectiveness of ECM can be degraded using electronic counter-countermeasures (ECCM) techniques $[2,4,6,7]$. One of the most robust ECCM methods against deception jamming is pulse diversity of radar signals, for example, multi-tone phase modulation and slowly varying chirp rate of linear frequency modulated (LFM) chirps are explored in [4]. Another method involves coding signals in such a fashion that a transmitted waveform at an arbitrary pulse repetition interval (PRI) will produce a low value of peak cross-correlation with the waveform at the previous PRI, thus severely limiting the effectiveness of deception jamming during the correlation process-one example of such an approach is random noise radar $[5,8-$ 11]. Orthogonal frequency division multiplexing (OFDM) can also be employed in this fashion; it is currently being implemented in multiple commercial communications systems [12-14], however its applications to radar have been somewhat limited to this day [15-20]. Advances in sampling 


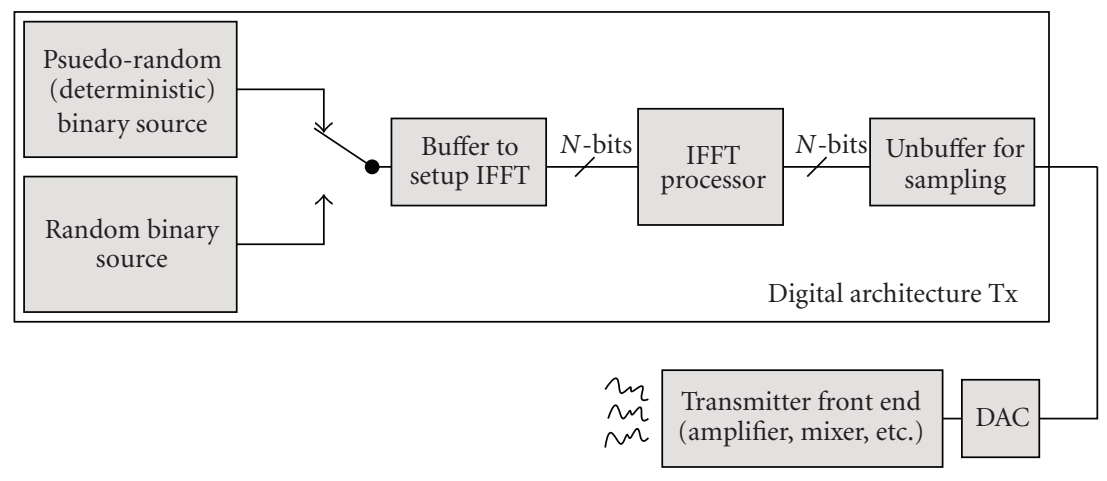

Figure 1: Block diagram of OFDM transmitter.

technology have made ultra-wideband (UWB) wave shaping a possibility for OFDM systems [21]. In our paper we contrast multifrequency UWB SAR signals based on OFDM with several common types of wideband SAR waveforms with certain similarities to OFDM. LFM chirps $[22,23]$, while easily implemented and widely used for SAR processing due to the relatively simple and efficient range-Doppler algorithm, experience high susceptibility to jamming because of the linear nature of the IF. Frequency-hopping (FH) signals $[24,25]$, similarly to OFDM, change spectral composition at each PRI, thus limiting the effectiveness of DRFM jammers. However, because their IF is constant, if the hopping interval is known, this type of signal may also be affected by IF jamming. On the other hand, ultra-short Gaussian monopulses [26], while allowing for submeter resolution and exhibiting good multipath performance, are usually processed using a different technique, backprojection, which is computationally expensive and varies significantly from range-Doppler processing employed in our simulations, thus these UWB SAR signals are not considered in the paper.

This paper investigates the ECCM capabilities of an UWB OFDM signal and the benchmark LFM chirp and FH signals against an IF jammer and a DRFM repeat jammer. Section 2.1 describes the OFDM signal model, Section 2.2 explores peak side lobe (PSL) and peak cross-correlation performance of wideband OFDM radar pulses with random subband composition, and Section 2.3 describes signal characteristics for the benchmark waveforms. Sections 3.1 and 3.2 describe deception jammer models for an IF estimator and a DRFM repeat jammer, respectively; Section 3.3 discusses how target image reconstruction is performed. Section 4 presents the simulation results, while conclusions are offered in Section 5.

\section{Signal Modeling and Characteristics}

2.1. OFDM Signal Construction. The analog baseband OFDM radar signal is given as

$$
s(t)=\operatorname{Re}\left(\sum_{k=1}^{N} x_{k} \cdot e^{j 2 \pi k(t / T)}\right), \quad 0<t<T,
$$

where $x_{k}$ is the $k$ th data symbol, $N$ is the number of subcarriers, and $T$ is the signal duration. The signal $s(t)$ is simply the sum of individual RF pulses known as subcarriers. Each subcarrier has a unique center frequency $k \Delta f$ where $\Delta f=1 / T$ is the frequency separation between each subcarrier. The individual spectra located at multiples of $\Delta f$ are known as subbands. Each $k$ th subcarrier has a corresponding subband which is described by a sinc function centered on $k \Delta f$. The subbands will overlap, but because of their orthogonality the peak of one subband will coincide with zeros for all other subbands. If we then sample $s(t)$ we can obtain the following baseband discrete time signal expression:

$$
s[n]=\sum_{k=1}^{N} x_{k} \cdot \cos \left(j 2 \pi k \cdot \Delta f \cdot \frac{T}{N}\right), \quad 0<t<T,
$$

where $T / N$ is the sampling rate, $1 / T$ has been replaced with $\Delta f$. The spectral characteristics of $s[n]$ are determined solely by the sampling rate of the D/A converter and the chosen number of subbands, $N$. Figure 1 shows a simplified block diagram of an OFDM transmitter.

A switch first determines whether a random or pseudorandom binary source will be used in generating the signal. The samples are sent from the source where they accumulate in an IFFT setup buffer. The buffer will eventually contain a vector representing the spectrum of the OFDM signal where each element is a subband of the signal. Recalling that the subcarriers are cosine waveforms it is advantageous to begin signal construction in the spectral domain by populating the vector with 1 's, 0 's, and -1 's to produce the correct spectra for the subcarriers. The $N_{s}$ samples are then sent along an $N_{s}$-bit bus to an IFFT processor where an inverse fast Fourier transform is performed on the digital frequency domain samples to obtain a discrete time domain representation of the OFDM signal. Another $\mathrm{N}_{s^{-}}$ bit bus passes the new time domain vector through an unbuffer and the elements are sent one at a time to the D/A converter to generate the analog OFDM signal. Similar to actual signal construction, simulation of an OFDM signal begins by randomly or pseudorandomly populating the digital frequency domain vector. The MATLAB format for filling a spectral domain vector is as follows: (1) the first 


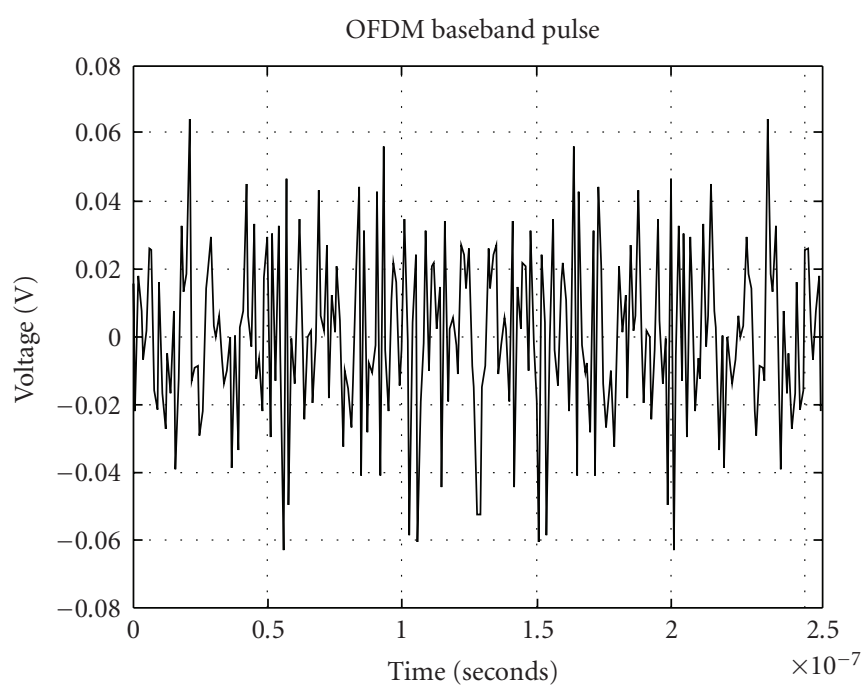

(a)

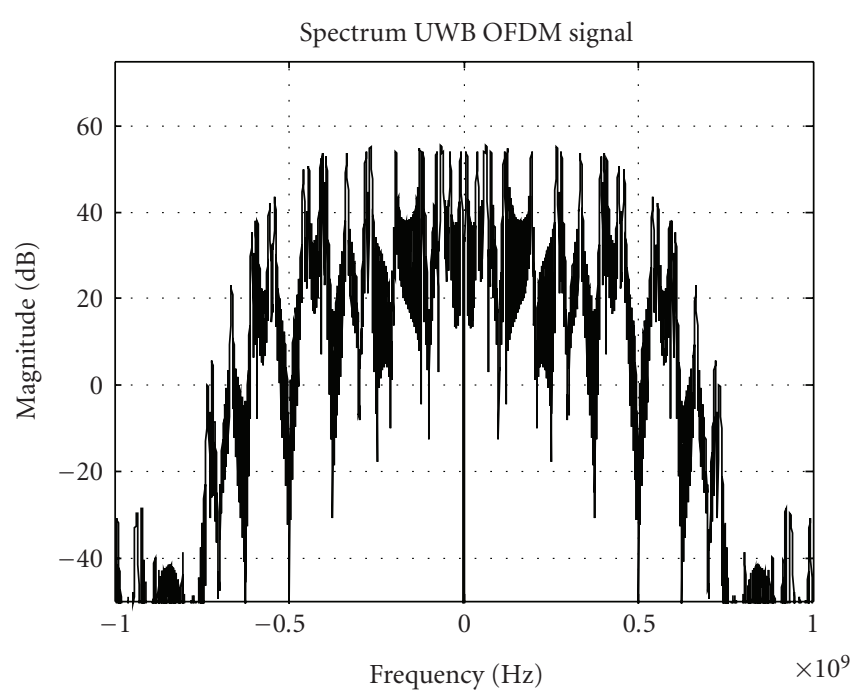

(b)

FIGURE 2: Simulated OFDM signal sampled at $1 \mathrm{Gs} / \mathrm{s}$ : (a) time domain signal, (b) signal spectrum.

element is the DC component and is zero in our case, (2) the positive half of the spectrum is added and (3) the negative half of the spectrum is added. The negative frequency block needs to be flipped before being added. An IFFT is performed on the frequency domain vector to create the simulated time domain OFDM signal. Figure 2(a) shows a simulated arbitrary OFDM signal sampled at rate of $1 \mathrm{Gs} / \mathrm{s}$ and Figure 2(b) shows the corresponding signal spectrum.

\subsection{Peak Side Lobe and Cross-Correlation Performance of} Random OFDM Signals. Radar ambiguity function (AF) is an important tool for understanding performance of a waveform. Conventionally, narrowband form of AF is used and closed-form integral solution for analog cases is obtained before plotting the function [23]. Such an approach, for example, was taken in [18] to plot AF of OFDM signal consisting of 8 subbands spread over $5 \mathrm{MHz}$ bandwidth. However, UWB OFDM signal should be treated differently due to its wide bandwidth. The error resultant from using narrowband approximation for computation of wideband signal's AF is derived and discussed in [19], which uses conventional integral format of AF definition. In [20] a similar approach is used to derive and optimize a narrowband $\mathrm{AF}$ of OFDM radar signals with up to 7 orthogonal subbands. In this work we derive the discrete form of UWB OFDM radar signal AF, as information extraction in OFDM system is performed on a digital baseband waveform - that is, all analysis below is based upon the down-converted receiver signal vectors sampled at the prescribed rate.

Normalized point target return for any type of wideband radar signal can be written as(3)

$$
s_{R X}(t)=s_{T X}(t-D(t)),
$$

where $s_{T X}$ is the transmit signal, $D(t)$ is roundtrip time delay. When target or radar platform (or both) is in motion, the roundtrip time delay $D(t)$ is a function of target range
$R(t)=R_{0}+v t$ where $R_{0}$ is point target's initial range and $v$ is the radial velocity, which is assumed to be constant during radar observation time; from [27],

$$
D(t)=\frac{2 R(t-(D(t) / 2))}{c}=\frac{2\left(R_{0}+v t\right)}{c+v},
$$

where $c$ is the velocity of light. Next, we need to convert (3) from general sample format into discrete time format; to translate sample indices into discrete time values we use $t_{k}=(k-1) \Delta t$, where $\Delta t$ is sampling interval, which is an inverse of D/A converter's sampling rate. This produces the expression for sampled transmit signal:

$$
\mathrm{s}_{T X}\left(t_{k}\right)=\sum_{n=1}^{N} x_{n} \cos \left(\frac{2 \pi n}{2 N+1}\left(\frac{t_{k}}{\Delta t}-N\right)\right), \quad k=1 \cdots 2 N+1 .
$$

Then, substituting (5) into (3) into it, we express sampled OFDM received signal as function of time and target's radial velocity, as shown in (6):

$$
\begin{aligned}
& s_{R X}\left(t_{k}, v\right) \\
& =\sum_{n=1}^{N} x_{n} \\
& \quad \times \cos \left(\frac{2 \pi n}{2 N+1}\left[\frac{((c-v) /(c+v)) t_{k}-\left(2 R_{0} /(c+v)\right)}{\Delta t}-N\right]\right) .
\end{aligned}
$$

Radar range profile reconstruction is performed via matched filtering. Instead of integral format, cross-correlation of sample-based data is a summation:

$$
\operatorname{MF}\left(\tau_{m}, v\right)=\sum_{k=1}^{2 N+1} s_{R X}\left(t_{k}, v\right) \cdot \hat{s}_{R X}\left(\tau_{m}-t_{k}\right),
$$


where $\widehat{s}_{R X}$ is the reference signal function, which is obtained from (6) by setting $v=0$.

Calculating absolute value of (7) and squaring it we recognize the result as a discrete AF of UWB OFDM radar signal:

$$
\chi\left(\tau_{m}, v\right)=\left|\operatorname{MF}\left(\tau_{m}, v\right)\right|^{2} .
$$

An example of thus simulated AF for the case of a $500 \mathrm{MHz}$-bandwidth OFDM pulse with 128 subbands with weights randomly selected from the set of $\{0,+1,-1\}$ and the total number of subbands with zero weight equal 17 is shown in Figure 3.

Due to the random nature of subband distribution, however, it is more beneficial to consider Monte Carlo simulations for an ensemble of realizations. In particular, when $s_{R X}$ and $\hat{s}_{R X}$ in (7) have the same underlying subband composition, a measure of peak side lobes (PSL) — which is defined as ratio of peak side lobe value to the peak main lobe value-can be obtained from the resultant zero-Doppler plots of an AF to evaluate range reconstruction performance. Conversely, when $s_{R X}$ and $\hat{s}_{R X}$ have different subband compositions, the cross-ambiguity function (XAF) will result from (8), which is a measure of orthogonality between two randomly generated OFDM pulses. PSL performance of a $500 \mathrm{MHz}$-bandwidth OFDM radar signal with random subband compositions, various numbers of subbands and subband fill ratios - defined as a ratio of nonzeroed subband number to the total number of subbands in a signal-is illustrated in Figure 4(a), whereas the plot of the maxima of XAFs for the case of $500 \mathrm{MHz}$-bandwidth and a total of 128 subbands is shown in Figure 4(b).

It is seen that with subband fill ratios above $50 \%$ and total number of subbands 128 or higher, OFDM signals exhibit better PSL performance than the benchmark samebandwidth LFM pulse (more on the benchmark pulse construction is in Section 2.3 below). It is also evident from the plot that if PSL is desired to be $\leq-20 \mathrm{~dB}$, a minimum number of subbands has to be 128 and a subband fill ratio greater than $80 \%$. In Figure 4(b) "inverse" scenario refers to the simplest method for cross-correlation minimization, which is to generate two OFDM pulses with the following spectral domain properties,

$$
\begin{aligned}
& \mathbf{S}_{1}= \begin{cases}S_{k 1}[n]= \pm 1, & \text { for } n \text { odd }, \\
S_{k 1}[n]=0, & \text { for } n \text { even, }\end{cases} \\
& \mathbf{S}_{2}= \begin{cases}S_{k 2}[n]=0, & \text { for } n \text { odd } \\
S_{k 2}[n]= \pm 1, & \text { for } n \text { even }\end{cases}
\end{aligned}
$$

Unfortunately, this method of signal generation is not optimal in practical applications, especially in jamming scenarios. Having only two unique signals dramatically increases the radar's susceptibility to deception jamming. Therefore, to penalize the jammer, it is essential that a radar system be capable of employing pulse diversity, while maintaining reasonable cross-correlation values. The ultimate pulse diversity can be achieved if the radar signal contains randomness, or, in the extreme case, bandlimited random noise can be used as a radar signal [5, 9-11]. Thus, if random OFDM subband distribution is assumed, it ensures transmission of a unique pulse at every PRI. As a comparison to other frequency-modulated schemes, in [24] it is noted, for example, that simultaneous minimization of AF everywhere except the point of origin (thus minimizing PSL) and XAF for every time-frequency point is challenging for frequency coded signals - signals with ideal AF (such as Costas arrays) will have poor CAF characteristics and vice versa - and the codes proposed by the authors of [24] exhibit maximum XAF peak of approximately $-6 \mathrm{~dB}$ compared to the AF peak value. For simulated UWB OFDM signal this level is reached at approximately $30 \%$ of subband fill ratio and it improves with higher fill ratios. If the second pulse is constructed as described in (9) we can obtain even better performance, reaching $-20 \mathrm{~dB}$ when the first pulse has nearly $90 \%$ subband fill ratio and, consequently, the second pulse has approximately $10 \%$ subband fill ratio. This, however, is a trade-off, as lowering the subband fill ratio for the second pulse to $10 \%$ will significantly degrade range resolution and ECCM characteristics of the radar, as discussed above.

\subsection{Benchmark Signals Construction. An LFM chirp can be expressed as}

$$
s_{\mathrm{lfm}}(t)=A_{\mathrm{lfm}} \exp \left[j \pi \cdot\left(2 f_{c}+k_{\mathrm{LFM}} t\right) \cdot t\right], \quad 0<t<T,
$$

where $A_{\text {lfm }}$ is the constant envelope used to equalize the energy, $f_{c}$ is the center frequency, $k_{\mathrm{LFM}}$ is the modulation rate, and $T$ is the duration of the chirp. Basic analog LFM transmitter implementation initially requires a pulsed sinusoid waveform at $f_{c}$ to be amplified and passed through an up/down-chirp filter. Using a passive surface acoustic wave (SAW) chirp filter as in [28] greatly reduces hardware complexity and decreases power needed in the transmitter design. The chirped pulse is passed through a power amplifier (PA) and transmitted through the antenna. The constant envelope (CE) waveform of the LFM chirp gives it high tolerances against nonlinearities requiring less stringent constraints in the PA.

An FH radar signal is given as

$$
\begin{array}{r}
s_{\mathrm{fh}}(t)=A_{\mathrm{fh}} \exp \left[j 2 \pi \cdot\left(f_{i}+f_{c}\right) \cdot t\right], \\
i=0,1,2, \ldots, 0<t<T,
\end{array}
$$

where $A_{\mathrm{fh}}$ is the constant used to equalize the energy, $f_{c}$ is the center frequency, and $f_{i}$ are the frequencies determined by pseudorandom selections of $i$ within a pre-determined range of frequencies. The transmitter consists of a clocked pseudorandom number $(\mathrm{PN})$ generator that sends a number $i$ to a frequency synthesizer which generates a sinusoid at $f_{i}$. The sinusoid is mixed with the carrier sinusoid at frequency $f_{c}$ to generate a sinusoidal waveform at $\left(f_{i}+f_{c}\right)$. The new waveform is band-pass filtered to eliminate the $\left(f_{i}-f_{c}\right)$ component acquired from mixing the two sinusoids. The waveform is then amplified and transmitted. Similar to the LFM chirp the FH signal is a CE-waveform and exhibits 


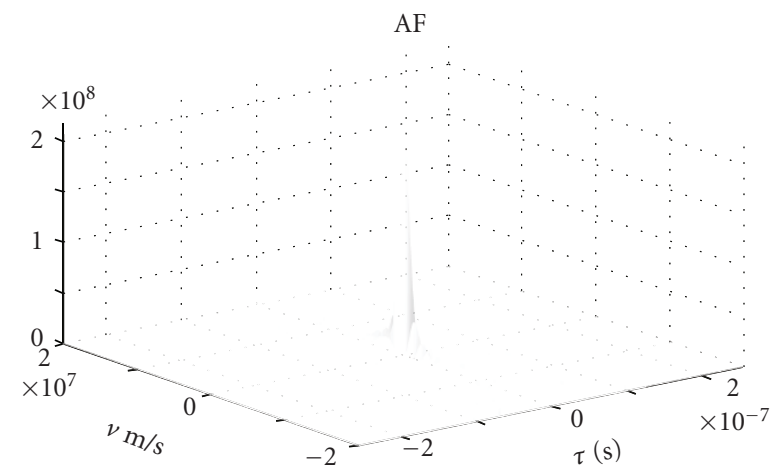

(a)

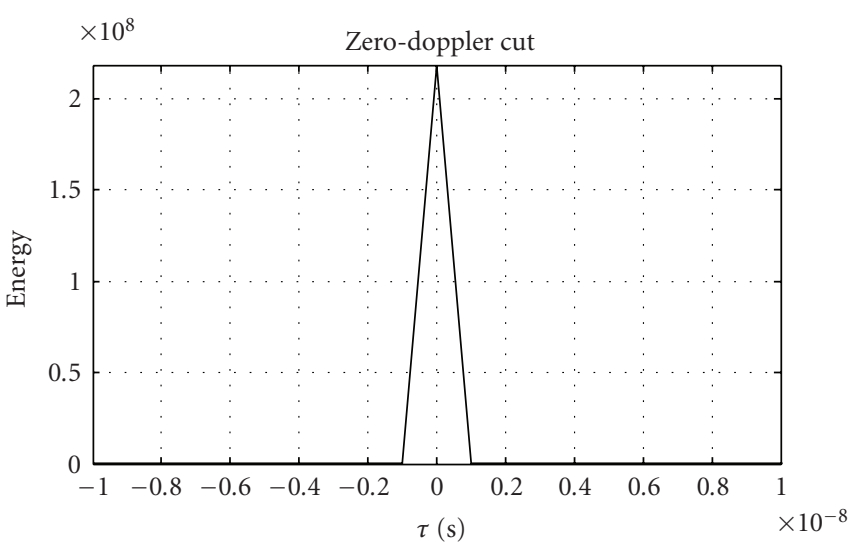

(c)

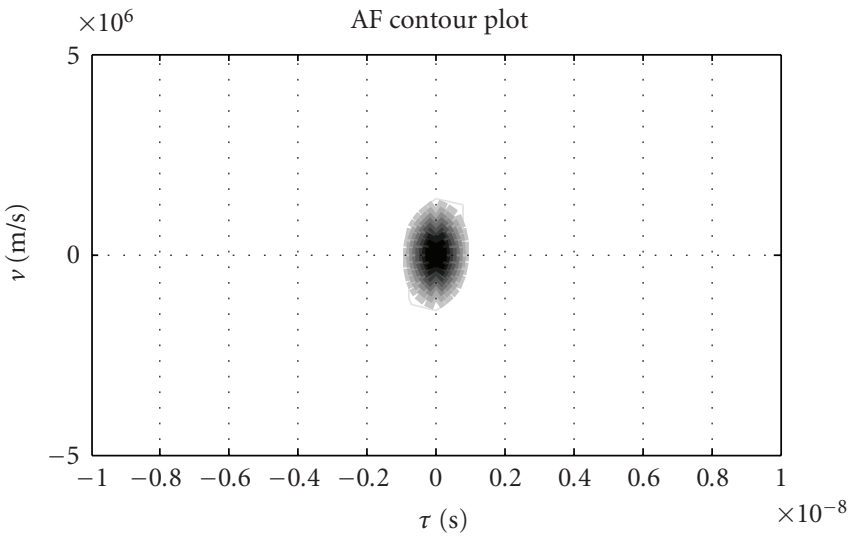

(b)

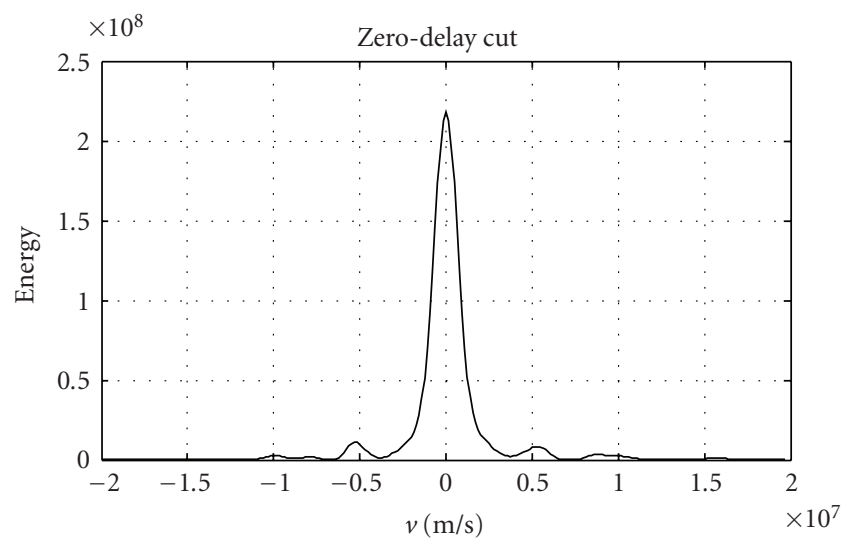

(d)

Figure 3: Example plot an UWB OFDM radar AF with random subband distribution: (a) 3-D plot of AF; (b) Contour plot of the center region; (c) Zero-Doppler cut; (d) Zero-delay cut.

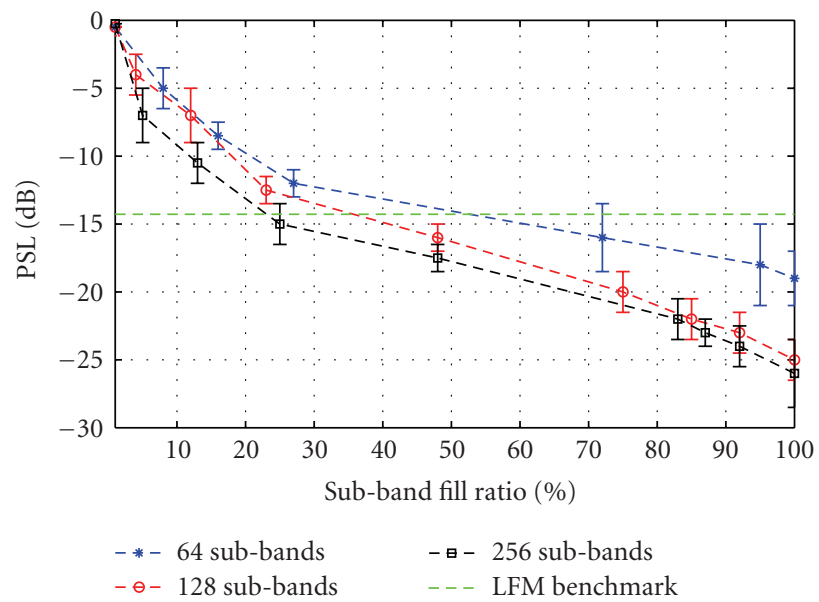

(a)

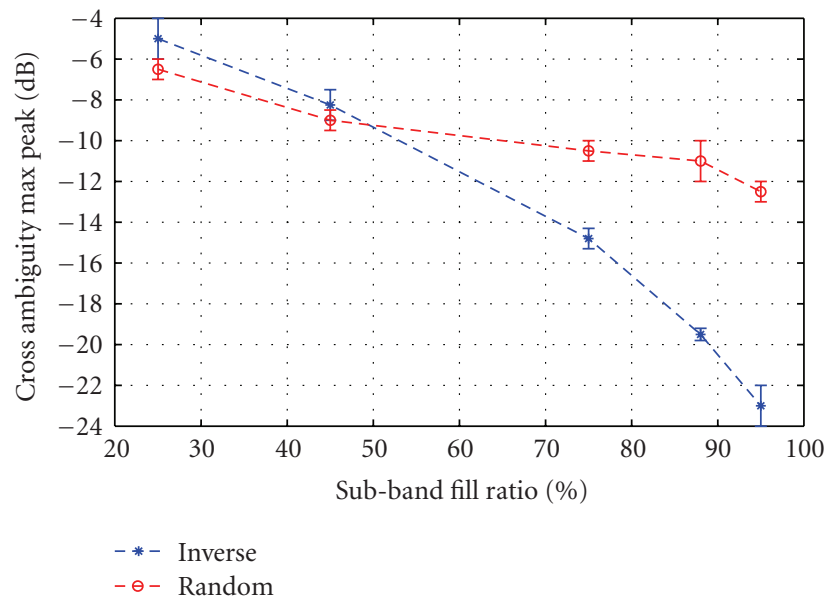

(b)

FIgUre 4: Monte Carlo simulation results for $500 \mathrm{MHz}$-bandwidth OFDM radar signals: (a) PSL performance; (b) Peak XAF performance for 128 subband case. 


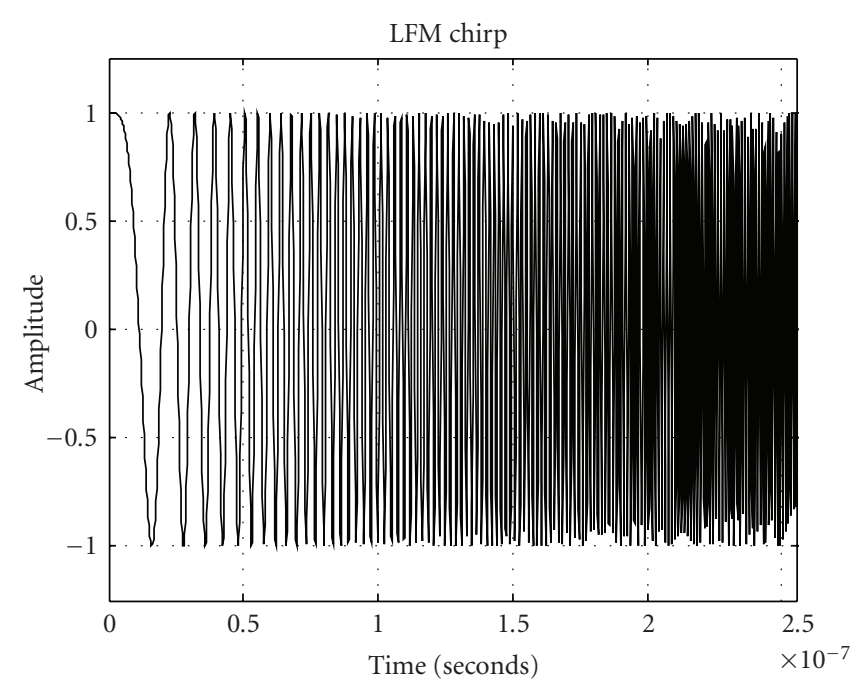

(a)

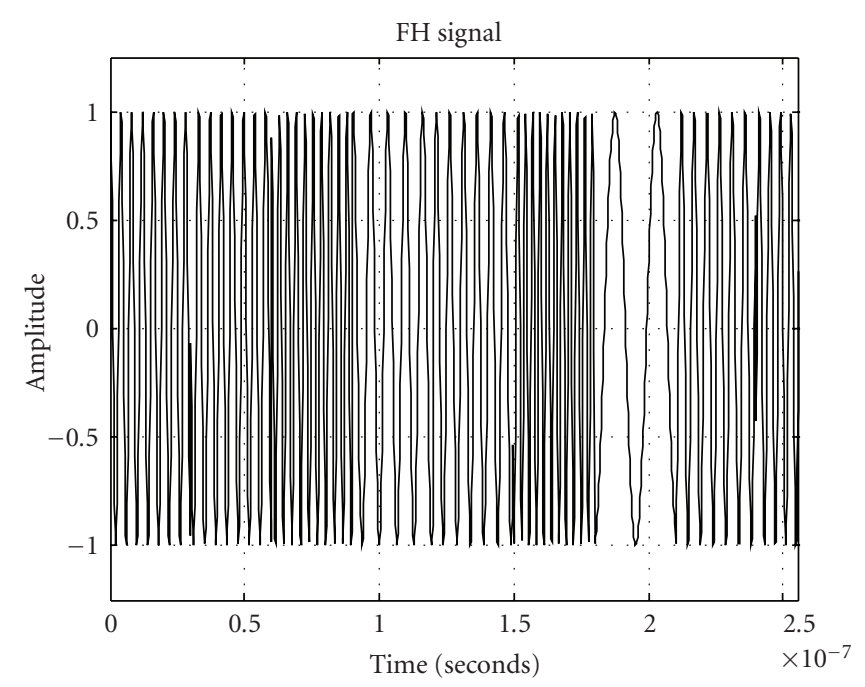

(c)

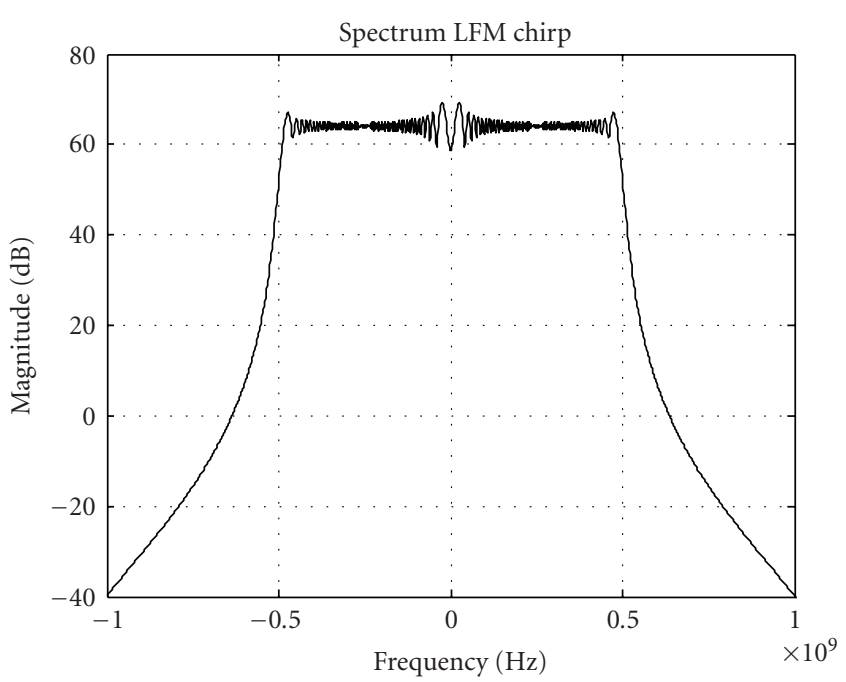

(b)

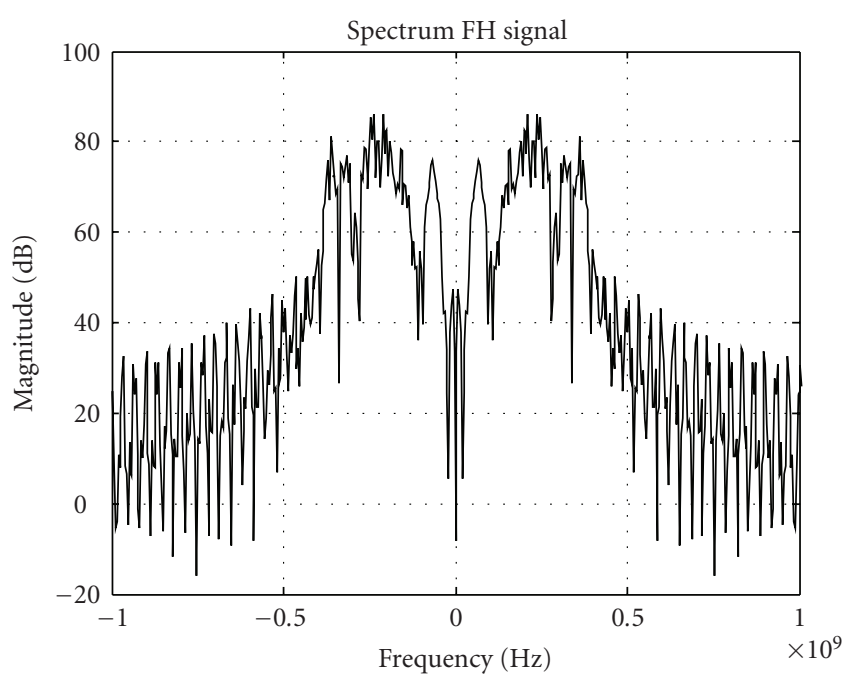

(d)

FIGURE 5: Benchmark signals: (a)-(b) LFM chirp and its spectrum; (c)-(d) FH signal and its spectrum.

the same high tolerances to nonlinearities in the amplifier. Figure 5 shows the time domain signals and corresponding spectrums for the benchmark waveforms.

Multifrequency characteristic of OFDM signals in comparison to the benchmark waveforms can be further illustrated via time-frequency analysis using spectrograms. An example of the spectrograms of the three types of radar signals is shown in Figure 6.

The graphs exhibit the differences between the three types of signals from the perspective of an intercepting entity. It is easy to see that the LFM signal's time-frequency behavior not only can be exactly inferred, but it can also be predicted. The tolerances in choosing appropriate time window lengths and shapes and sampling frequency are very wide and it is intuitive that the clarity of the analysis will remain the same for a number of chirps-not just LFM, but also nonlinear FM chirps, such as quadratic, logarithmic, and so forth, Thus, qualitatively, FM chirp will require the least time for the intercepting jammer to analyze and reproduce the signal. The second signal-FH pulse-is admittedly more difficult to reproduce and predict, as its time-frequency representation does not follow any mathematical function. However, knowing the hop interval and starting point of the pulse we can choose the time window so that it coincides with the hop interval, providing for the graph shown in Figure 6. Locating $y$-axis maxima within each time window then clearly shows that we can, indeed, recover time-frequency portrait of an $\mathrm{FH}$ signal - white dots overlaid on top of the spectrogram graph represent both the locations of maxima and the original values of hop frequencies in the FH signal. Of course, there are limitations to this perfect-case scenario: the interceptor is required to know the hop interval duration - which must be constant within a pulse-and oversampling of the received signal is required to ensure quality capture of the signal 

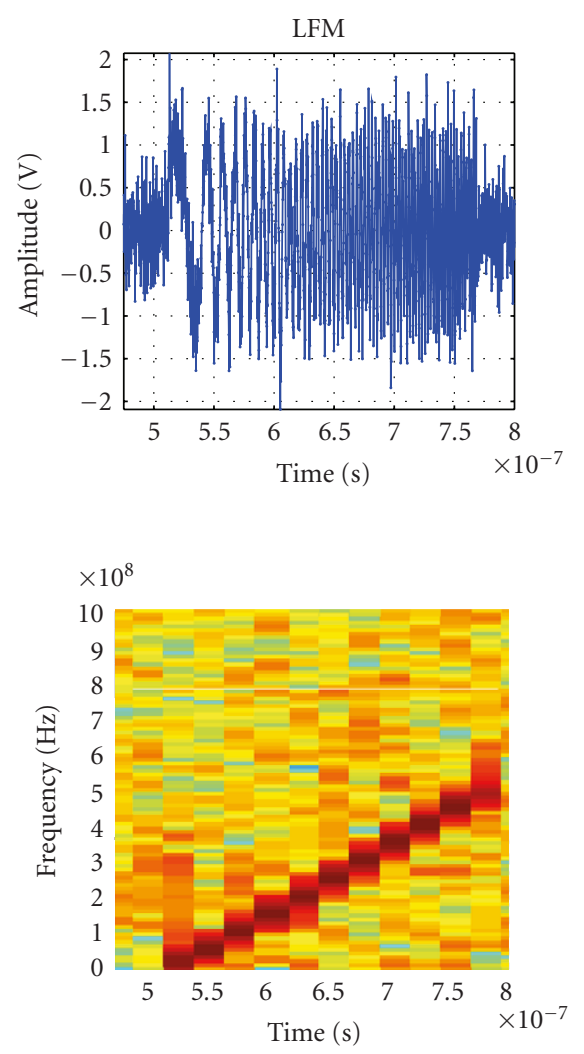

(d)

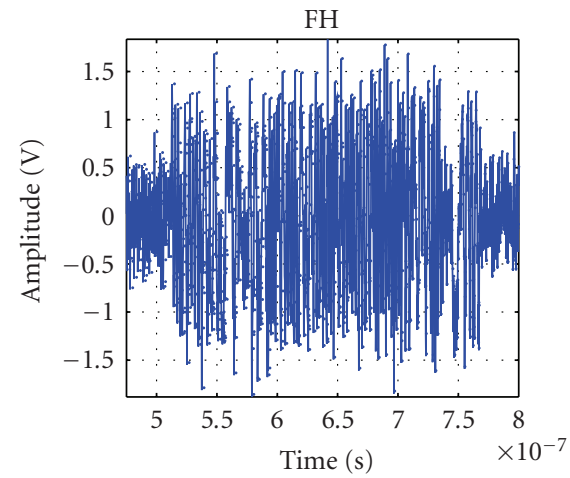

(b)

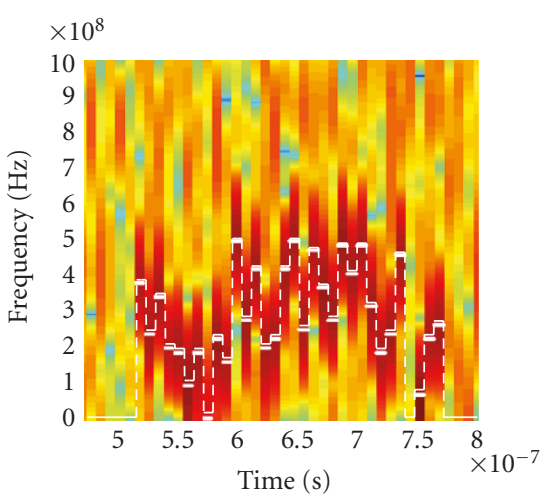

(e)

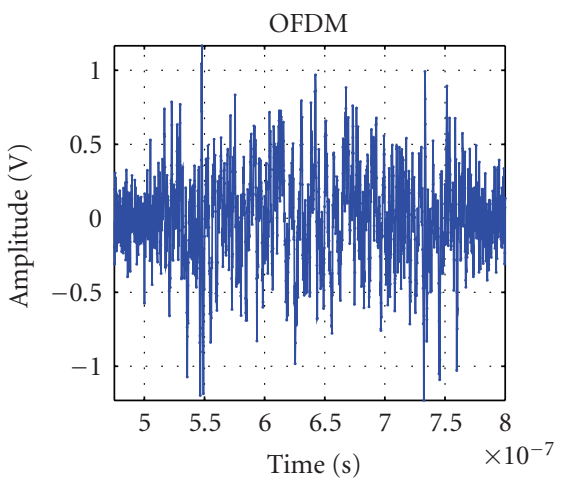

(c)

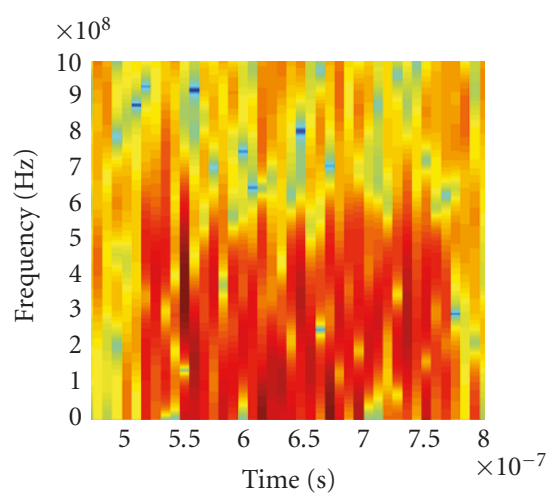

(f)

Figure 6: Time-frequency representation of the three types of SAR signals: LFM, FH and OFDM.

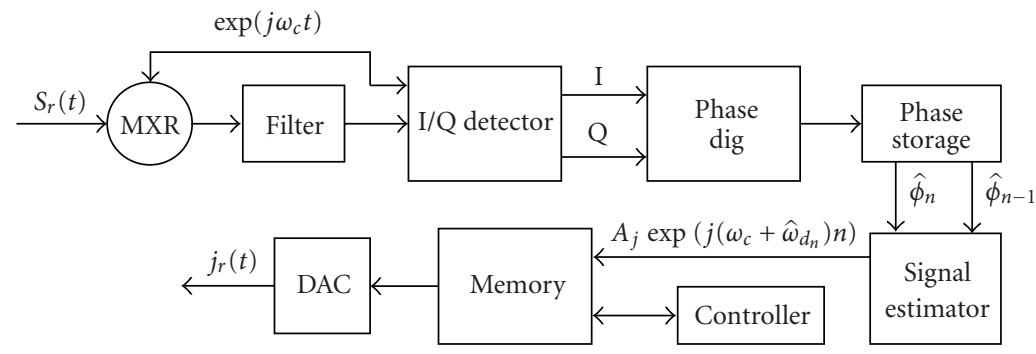

FIGURE 7: Block diagram of an instantaneous frequency deception jammer.

within each hop interval (in our case we collect 40 samples per hop interval of $8 \mathrm{~ns}$ ). The third signal-OFDM pulseis evidently the hardest to intercept and predict. In fact, its uniqueness is such that no amount of oversampling and no size of a fractional sample window will allow the interceptor to resolve the time-frequency characteristics of an OFDM signal precisely. This is due to the fact that the signal is inherently multifrequency.

\section{Deception Jammer Model Implementation}

3.1. Instantaneous Frequency (IF) Estimator. The analytic signal $z(t)$ is given as

$$
z(t)=A(t) \exp [j \phi(t)]
$$

where $A(t)$ is the time dependent amplitude and $\phi(t)$ is the phase function of the real signal. The IF of (12) has commonly been defined as

$$
\widehat{\omega}(t)=\frac{d \phi(t)}{d t}=\phi^{\prime}(t) .
$$

However, examples given in $[29,30]$ show that (13) may not be a suitable definition in all cases, particularly the case of multi-component signals. It was stated in [29] that (13) will give physically meaningful results only if the spectrum of the signal is symmetric about a center frequency. The UWB OFDM signal, LFM chirp, and FH signal all exhibit this characteristic and, therefore, (13) is sufficient for determining the IF of the waveforms.

A block diagram of an IF deception jammer is shown in Figure 7; it is assumed that the center frequency of 
the intercepted radar signal is known to the jammer. An arbitrary signal, $s_{r}(t)=A_{r} \exp \left[j\left(\omega_{c} t+\phi(t)\right)\right]$ is first mixed with a carrier at the known center frequency and filtered to remove undesirable components to obtain $s_{\text {mixed }}(t)=$ $\exp \left[j\left(\omega_{c} t+\phi(t)-\omega_{c} t\right)\right]=\exp [j \phi(t)]$ which is simply a complex exponential containing the phase information of the intercepted waveform. The signal is sent through an I/Q detector to recover the in-phase and quadrature components, $\mathrm{I}=\cos (\phi(t))$ and $\mathrm{Q}=\sin (\phi(t))$. The I/Q channels are fed into a phase digitizer that determines the discrete instantaneous phase $\hat{\phi}_{n}$ from the sampled $I_{n} / Q_{n}$ inputs and then each $\hat{\phi}_{n}$ is stored. A signal estimator block is then used to generate the discrete waveform,

$$
j_{r}[n]=A_{j} \exp \left(j\left(\omega_{c}+\widehat{\omega}_{d_{n}}\right) n\right)=A_{j} \exp \left(j \widehat{\omega}_{n} n\right),
$$

where $\widehat{\omega}_{n}=\left(\omega_{c}+\widehat{\omega}_{d_{n}}\right)$ is the discrete IF of the waveform and $\widehat{\omega}_{d_{n}}$ is the discrete IF deviation and is expressed as

$$
\widehat{\omega}_{d_{n}}=\frac{\hat{\phi}_{n}-\hat{\phi}_{n-1}}{T_{s}}
$$

which is the discrete derivative calculated by using the current and previous instantaneous phases along with the time sampling interval $T_{s}$. The discrete waveform is delayed to give the signal a false range offset and stored in memory. At the next PRI the discrete waveform is sent through a D/A converter and transmitted in the final form,

$$
j_{r}(t)=A_{j} \exp \left(j\left(\omega_{c}+\widehat{\omega}_{d}(t)\right) t\right)=A_{j} \exp (j \widehat{\omega}(t) t),
$$

where $\widehat{\omega}(t)=\omega_{c}+\widehat{\omega}_{d}(t)$ and $j_{r}(t)=A_{j} s_{r}\left(t-t_{d}\right)$ with $t_{d}$ being the time delay (range offset) of the signal.

The IF expressions for the LFM chirp, OFDM pulse and $\mathrm{FH}$ signal were derived in [31] and are given below:

$$
\begin{gathered}
\hat{\omega}_{\mathrm{lfm}}(t)=2 \pi k_{\mathrm{LFM}} t+\omega_{c}, \\
\hat{\omega}_{\mathrm{ofdm}}(t)=\frac{d}{d t}\left[\arctan \left(\frac{\gamma_{r} \sin (2 \pi N \Delta f t)}{\gamma_{r} \cos (2 \pi N \Delta f t)-1}\right)\right], \\
\hat{\omega}_{\mathrm{FH}}=2 \pi \cdot f_{i}+\omega_{c},
\end{gathered}
$$

where $2 \pi k_{\mathrm{LFM}} t=\widehat{\omega}_{d_{\mathrm{ffm}}}(t)$ is the instantaneous frequency deviation of the LFM chirp, and $\gamma_{r}=\left(\left(1-e^{j 2 \pi(N+1) \Delta f \cdot t}\right) /(1-\right.$ $\left.\left.e^{j 2 \pi \Delta f \cdot t}\right)\right) \cdot e^{-j 2 \pi N \Delta f \cdot t}$. Comparing (16) to (17) we see that the IF generated by the jammer precisely matches the theoretically defined IF for the LFM chirp, whereas (16) and (18) will not match. It is important to note that the frequency hopping interval $\tau_{\mathrm{FH}}$ determines $f_{i}$ at any given time $t$ and is crucial in determining the IF of the $\mathrm{FH}$ signal-we assume that $\tau_{\mathrm{FH}}$ is known to the jammer.

3.2. DRFM-Based Architecture. A block diagram of a DRFM repeat jammer is shown in Figure 8. A local oscillator generates a carrier signal at the known center frequency that is then mixed with the intercepted radar signal $s_{r}(t)$ $=A_{r} \exp \left[j\left(\omega_{c} t+\phi(t)\right)\right]$. The baseband waveform enters an $\mathrm{A} / \mathrm{D}$ converter where it is sampled at the sampling interval $T_{s}$ to produce the discrete signal $s_{\text {mixed }}[n]=\exp \left[j \phi_{n} n\right]$.
An unavoidable product of sampling is signal quantization error, which, if large enough can yield unacceptable results when generating replica waveforms. However, higher bit resolution reduces sampling speed subsequently reducing the instantaneous bandwidth of the DRFM jammer. A delay is introduced to the discrete signal creating a false range offset by means of a controller and the delayed signal is stored in memory until the next predicted PRI. The discrete delayed signal $j_{r}[n]=\exp \left[j \phi_{n}\left(n-n_{d}\right)\right]$ passes through a D/A converter and is mixed with an exponential at the known center frequency resulting in the transmitted jammer signal which can be expressed as

$$
j_{r}(t)=A_{j} s_{r}\left(t-t_{d}\right)
$$

where $t_{d}$ is the time delay of the signal.

DRFM jammer simulation will consist of copying and delaying the complete radar signal by a certain time period to introduce the false range offset. It is assumed that the jammer is capable of producing an exact replication of the intercepted radar signal.

3.3. Target Image Construction. The reconstructed radar image is formed based on range and cross-range profiles of the target area. Figure 9 shows an example of a SAR scenario, in which the SAR platform is moving along a straight trajectory and illuminates the target scene consisting of a number of point targets (strong reflectors) by emitting a signal at each $y=u_{k}$ position. Each target will reflect the signal back to the radar receiver with an introduced time delay and phase shift that depends on the position of the target. SAR signal processing methods are then used to generate range and cross-range profiles which determine target position.

Both range and cross-range profile reconstructions are achieved via matched filtering, but the domain crosscorrelation is performed in is slow-time-as opposed to range reconstruction, performed in fast-time (terminology and approach are per [32]). Following derivation of spherical phase-modulated (PM) signal within limited synthesized aperture [32] representing return signal phase characteristic along the cross-range coordinate $y$ as shown in Figure 9, we express the single-frequency return signal in slow-time domain as

$$
s_{R X S T}\left(\omega_{0}, y\right)=\sum_{n} \sigma_{n} \exp \left[-j 2 \frac{\omega_{0}}{c} \sqrt{x_{n}^{2}+\left(y_{n}-y\right)^{2}}\right],
$$

where $\omega_{0}$ is a frequency of the single-frequency component of the radar signal and $\sigma_{n}$ is $n$th point target reflectivity coefficient.

It can be seen that implementing (21) for a general UWB signal would require consideration of individual frequency components within the spectrum of such a signal. This can be achieved for the case of OFDM signals by considering a single subband as an approximation for a single-frequency component. Indeed, selecting $k$ th subband of the UWB OFDM signal as a reference, we can generate the phase response of an ideal point target located at the origin of cross-range coordinate by stepping through radar positions 


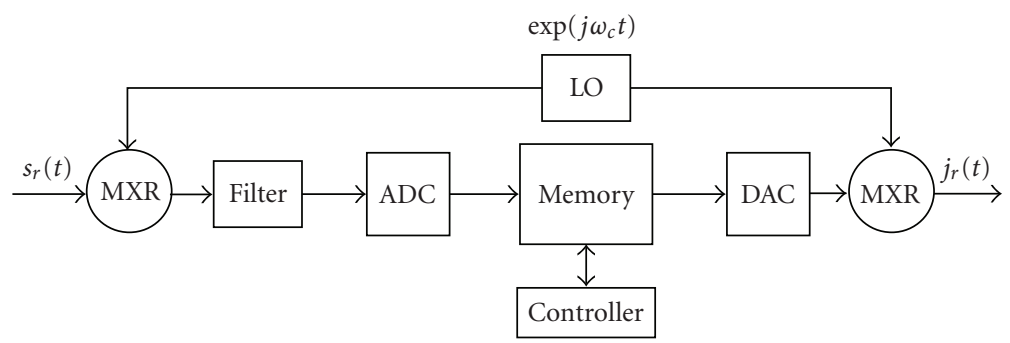

FIGURE 8: Block diagram of DRFM repeat jammer.

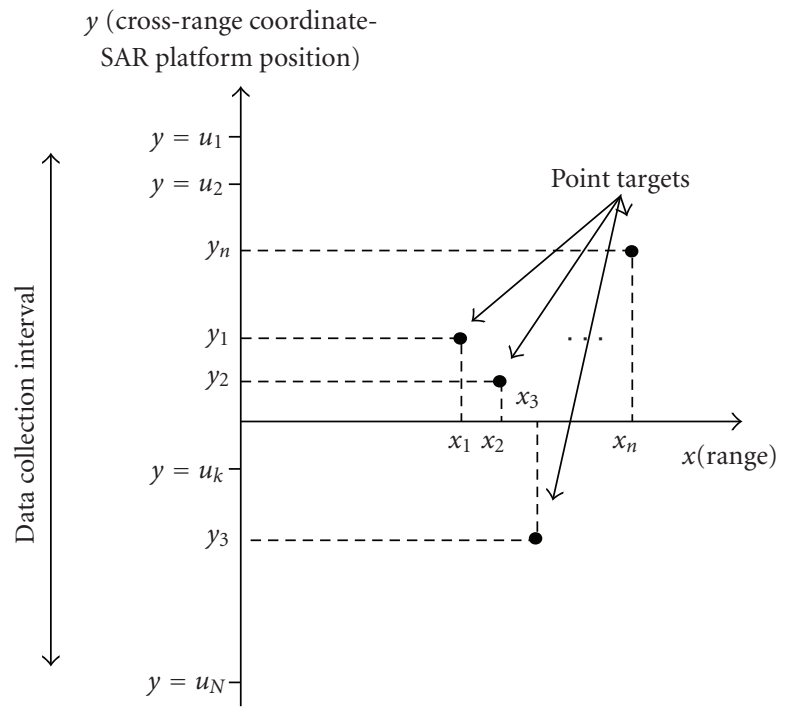

FIGURE 9: An illustration of a stripmap SAR scenario.

$y$ for the center frequency $\omega_{k}$ of this subband. Cross-range reconstruction can then be obtained by matched filtering of the actual phase history function for the same subcarrier $\omega_{k}$ and the reference phase response. Example of such a reference function generated for a cross-range swath $(-40$, 20) meters, as well as the simulated phase history of a point target located at +10 meters in cross-range and the resultant cross-range profile reconstruction are shown in Figures 10(a)-10(c), respectively.

The dependence of (21) on radar position demands that the jammer have the ability to accurately predict the PRI to ensure pulse-to-pulse phase coherence between transmitted jammer signals - otherwise, the jammer and radar reference phase function will be poorly correlated and the false target will not appear in the resulting reconstructed image. The jammer echoed signal must have the form

$$
j_{r}(t, y)=\sigma_{j} s_{r}\left(t-\frac{2 \sqrt{x_{j}^{2}+\left(y_{j}-y\right)^{2}}}{c}\right)
$$

where the $\sigma_{j}$ is the reflectivity coefficient of the false target and $x_{j}, y_{j}$ are the range and cross-range of the false target, respectively, as designated by the jammer. It is also evident from (22) that radar transmit position $y$ must be known to the jammer. Thus, the generated jammer signals for the OFDM, LFM chirp, and FH waveform are given, as in [31],

$$
\begin{aligned}
& j_{\mathrm{ofdm}}(t, y) \\
&=\sum_{k=1}^{N} \sigma_{j} \exp \left[j 2 \pi k\left(f_{c}+\Delta f\right)\left(t-\frac{\sqrt{x_{j}^{2}+\left(y_{j}-y\right)^{2}}}{c}\right)\right], \\
& j_{\mathrm{lfm}}(t, y) \\
&=\sigma_{j} \exp \left[j \pi\left(2 f_{c}+k_{\mathrm{LFM}}\left(t-\frac{\sqrt{x_{j}^{2}+\left(y_{j}-y\right)^{2}}}{c}\right)\right)\right. \\
&\left.\times\left(t-\frac{\sqrt{x_{j}^{2}+\left(y_{j}-y\right)^{2}}}{c}\right)\right]
\end{aligned}
$$

$j_{\text {fh }}(t, y)$

$$
=\sigma_{j} \exp \left[j 2 \pi\left(f_{i}+f_{c}\right)\left(t-\frac{\sqrt{x_{j}^{2}+\left(y_{j}-y\right)^{2}}}{c}\right)\right] .
$$




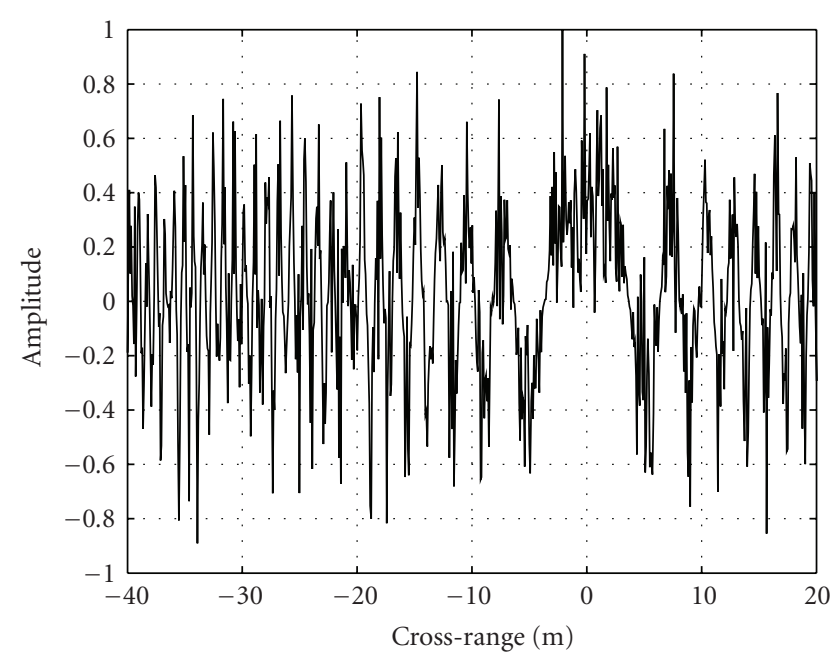

(a) Reference phase history

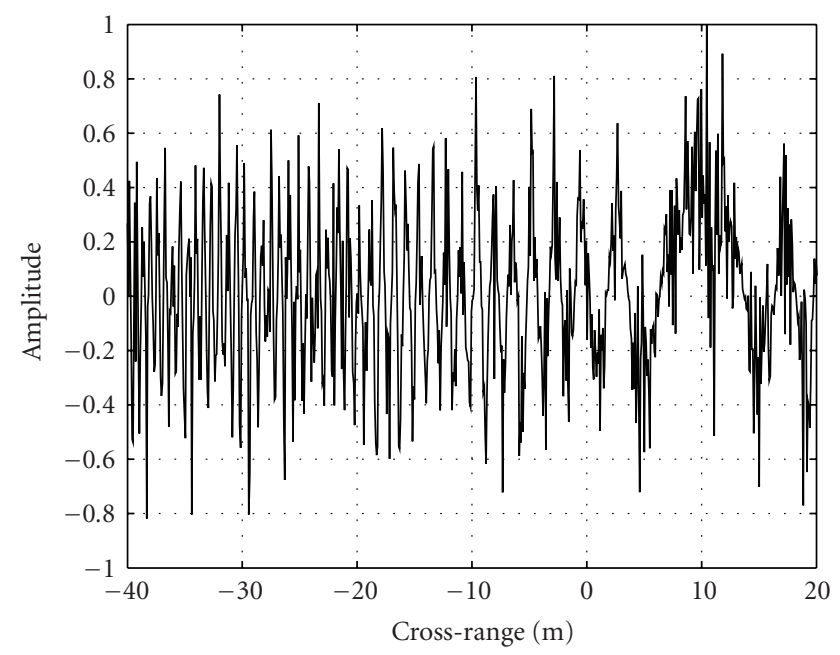

(b) Received phase history

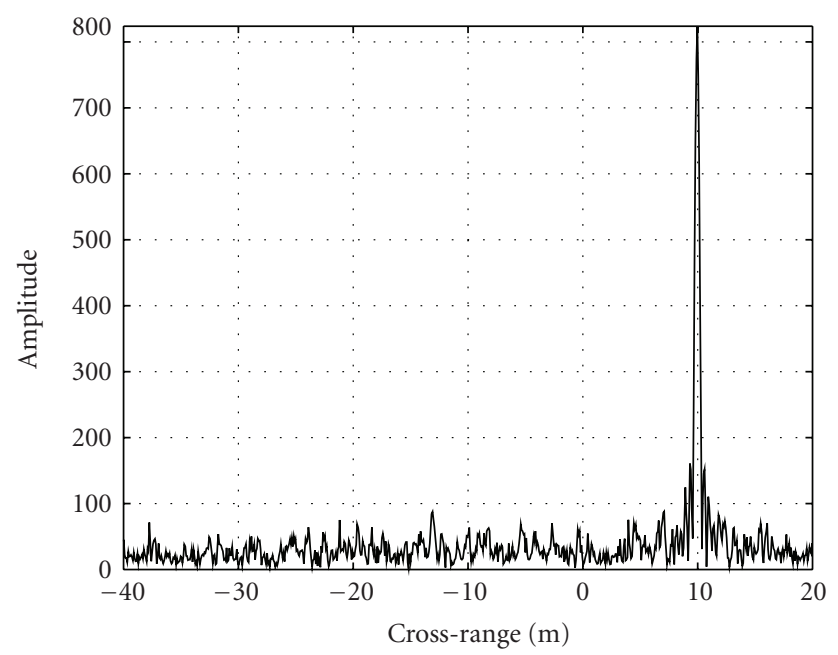

(c) Cross-range profile

FIgURE 10: Cross-range reconstruction using a single subband of an UWB OFDM pulse: (a) Reference phase history function, (b) simulated received phase history, (c) reconstructed cross-range profile.

\section{False Image Simulation Results}

Next, both IF and DRFM jammer signals were generated and used in deception jamming simulations with all three SAR waveforms: OFDM, LFM and FH. The LFM chirp with constant amplitude of 1 served as the baseline signal with respect to energy. Since the OFDM and FH waveforms used in simulation were randomly generated at each PRI, average energies of both signals were used when determining their amplitudes, which were adjusted to match the baseline energy figure. All SAR signals' baseband bandwidths were set to $0.5 \mathrm{GHz}$, and the sampling frequency of the jammer was set to $5 \mathrm{GHz}$. For all signals the same pulse duration of $256 \mathrm{~ns}$ was used. Signal-to-jammer ratio (SJR) was approximately $0 \mathrm{~dB}$ for all simulations. Figure 11 shows the simulated reconstructed images when the radar is in the presence of an IF jammer and a DRFM repeat jammer, which attempted to create an extended false target image at 130 meter in range and 10 to 25 meters in cross-range, whereas the real extended target was located also at 130 meters of range, but -25 to -10 meters in cross-range. The signals created by the IF jammer and the DRFM jammer had weak correlation with the transmitted OFDM signal subsequently causing the false target ranges to be nonexistent in the range profile. Therefore, the absence of a false target in Figures 11(a) and 11(b) is not unexpected. Further examining Figure 11 we can clearly see that the LFM chirp had strong correlation with both jammer signals causing false target ranges to be present in the generated range profiles. Figures 11(c) and 11(d) show that there are false targets in both reconstructed images. Since the FH signal varies at every PRI, the waveform recorded and retransmitted by the DRFM signal at the current PRI will always have weak correlation with the transmitted signal at all successive PRI's resulting in the absence of the false target in Figure 11(f). If the IF jammer can replicate the FH signal it will allow for strong correlation and cause a false target to be present in the reconstructed image, as shown in Figure 11(e). These results show that OFDM signals had the best overall performance against IF jammer and the DRFM jammer.

\section{Conclusion}

A multifrequency UWB OFDM signal model was developed and compared with two common radar imaging transmit signals, the LFM chirp and FH signal. A discrete AF of an UWB OFDM signal with random subband distribution was used to obtain PSL and cross-correlation performance characteristics in radar imaging scenarios. It was established that for a $500 \mathrm{MHz}$-bandwidth OFDM signal the minimum number of subbands and subband fill ratio required to exceed the performance of a same-bandwidth LFM chirp were 128 and 80\%, respectively. Random spectral composition of such a signal ensures strong ECCM capabilities in presence of deception jamming, at the resultant PSL and peak cross-correlation values of approximately $-22 \mathrm{~dB}$ and $-12 \mathrm{~dB}$, respectively. IF and DRFM jammers were modeled and used to introduce false targets into the imaging area of the radar system at $\mathrm{SJR} \approx 0 \mathrm{~dB}$. The two jammer models tested pulse diversity of each transmit signal, specifically 


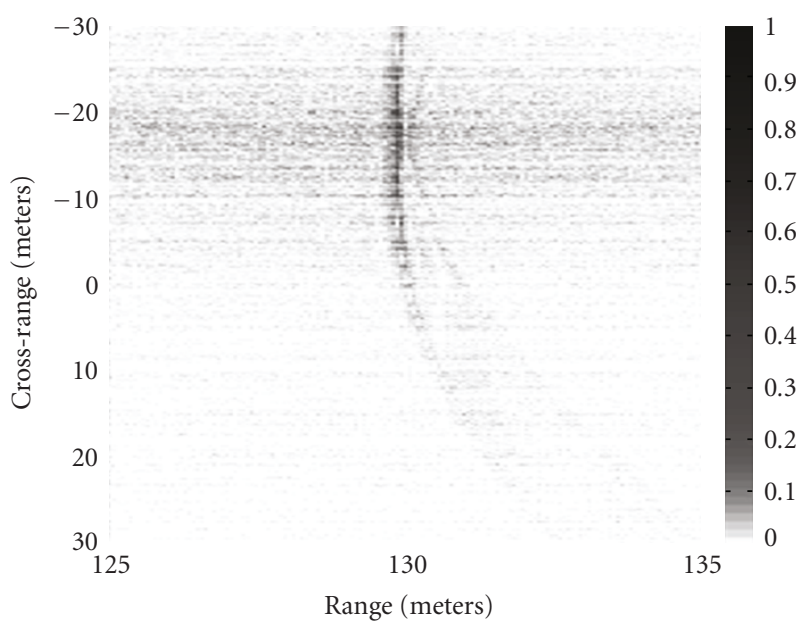

(a) Simulated image using OFDM signal with IF jammer

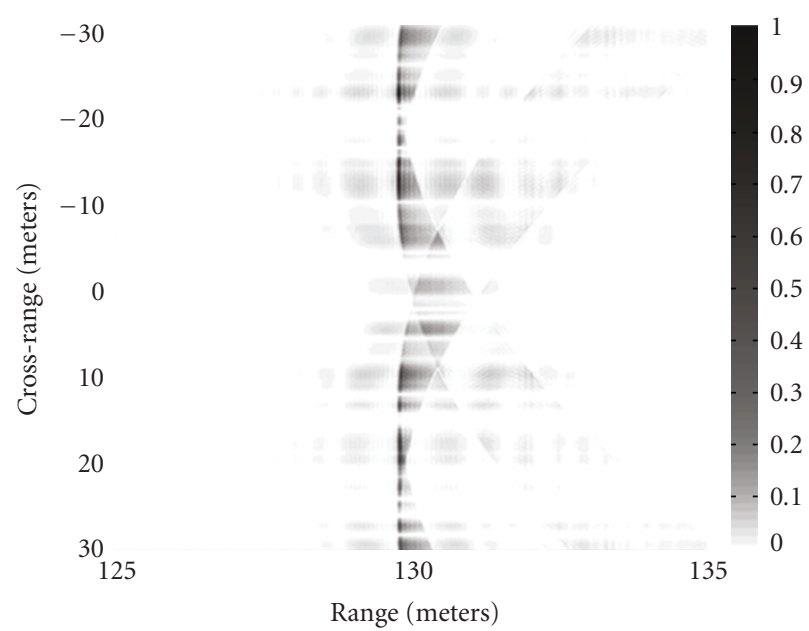

(c) Simulated image using LFM chirp with IF jammer

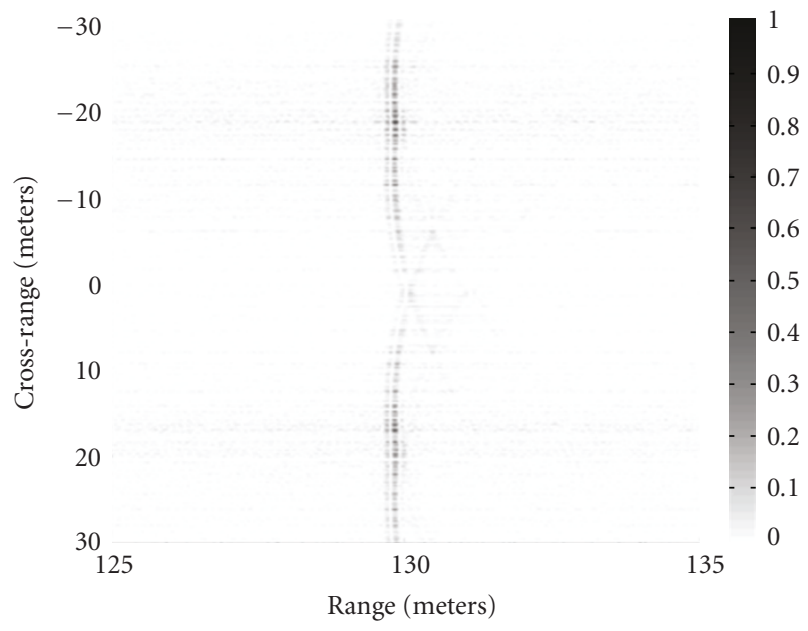

(e) Simulated image using FH signal with IF jammer

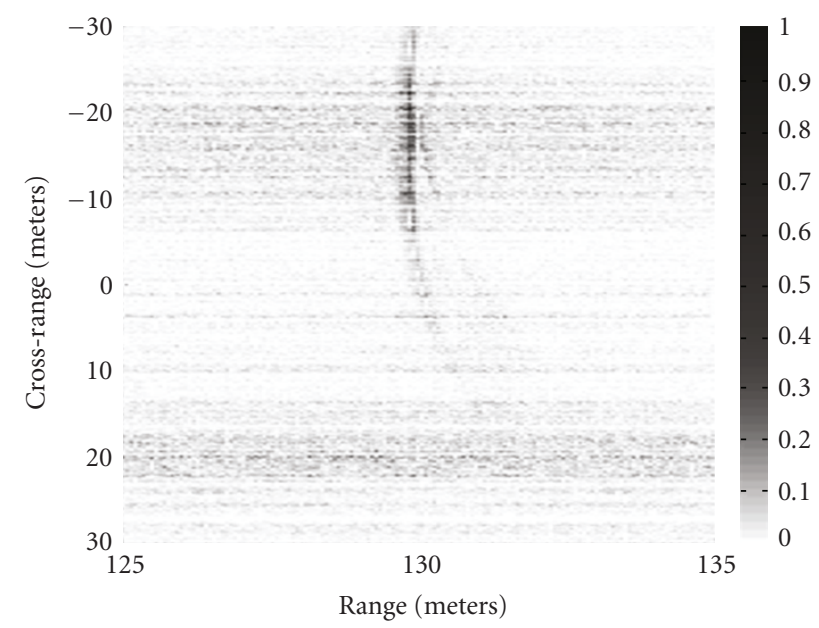

(b) Simulated image using OFDM signal with DRFM jammer

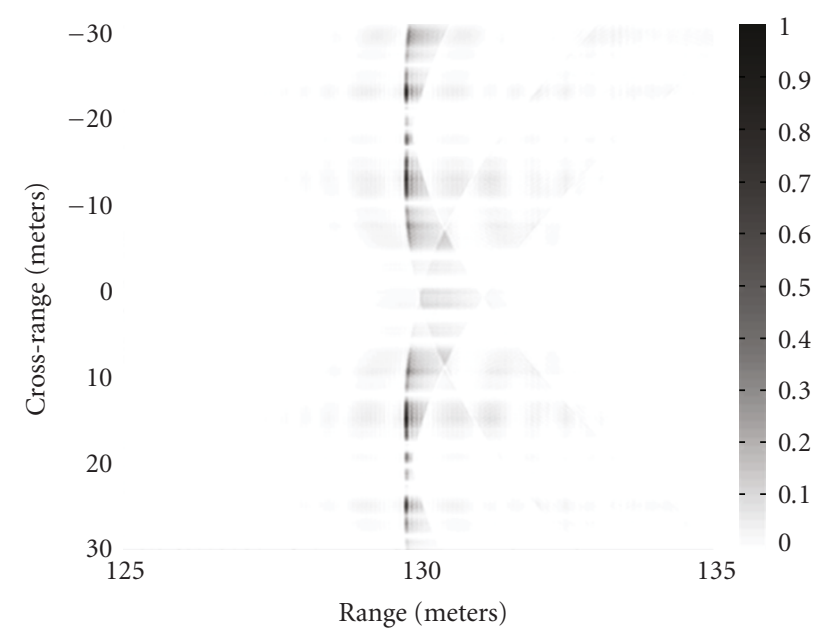

(d) Simulated image using LFM chirp with DRFM jammer

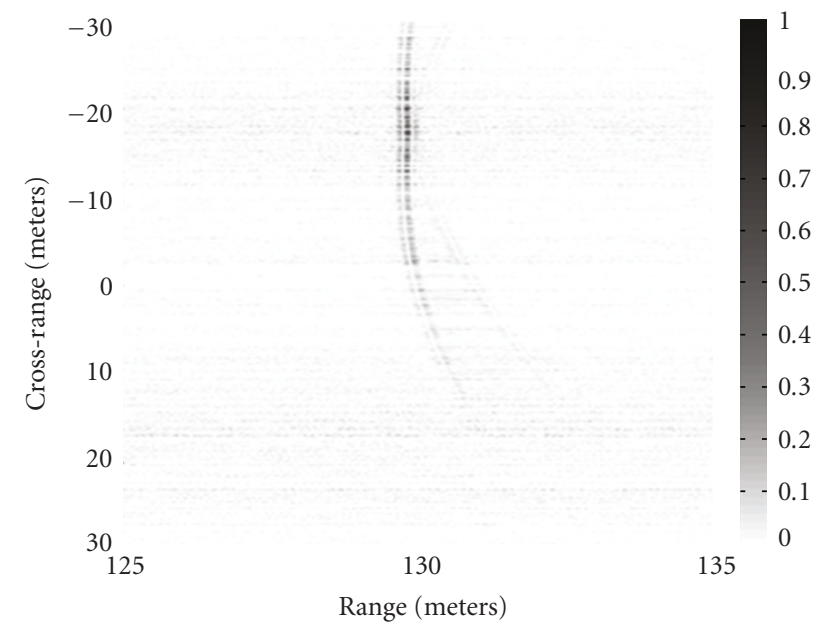

(f) Simulated image using FH signal with DRFM jammer

FIGURE 11: Simulated reconstructed images with jammer false targets: (a) OFDM signal with IF jammer; (b) OFDM signal with DRFM jammer; (c) LFM chirp with IF jammer; (d) LFM chirp with DRFM jammer; (e) FH signal with IF jammer; (f) FH signal with DRFM jammer. 
frequency diversity, and the ability of each signal to suppress jammer waveform effects during image reconstruction. The appearance of false targets in both jamming scenarios for the common LFM chirp clearly demonstrates the lack of ECCM capabilities. The frequency agility of both the OFDM and FH signals proved useful in the DRFM repeat jammer scenario. Although the jammer could produce replicas of the signals, the orthogonality of the waveforms from adjacent transmission intervals amounted to weak correlation between jammer and radar signals. If the IF jammer knows the hop interval of a FH signal, false target image introduction will result-however, due to the spectral structure of the OFDM signal, no false target images are found in OFDM SAR image simulations. These qualities make UWB OFDM waveforms with random subband distribution a viable choice for usage in SAR scenarios with deception jamming.

\section{Acknowledgments}

The authors are grateful to the anonymous reviewers, whose valuable and thorough suggestions have resulted in the much improved paper. They also wish to thank Dr. Jon Sjogren of AFOSR for project support and program reviews. This work was supported by the U.S. Air Force Office of Scientific Research under Grant FA9550-07-1-0297.

\section{References}

[1] M. Soumekh, "Reconnaissance with ultra wideband UHF synthetic aperture radar," IEEE Signal Processing Magazine, vol. 12, no. 4, pp. 21-40, 1995.

[2] J. D. Mosinski, "Electronic countermeasures," in Proceedings of the Tactical on Communications Conference, vol. 1, pp. 191195, Fort Wayne, Ind, USA, 1992.

[3] N. Liu and Y. Zhang, "A survey of radar ECM and ECCM," IEEE Transactions on Aerospace and Electronic Systems, vol. 31, no. 3, pp. 1110-1120, 1995.

[4] M. Soumekh, "SAR-ECCM using phase-perturbed LFM chirp signals and DRFM repeat jammer penalization," IEEE Transactions on Aerospace and Electronic Systems, vol. 42, no. 1, pp. 191-205, 2006.

[5] D. S. Garmatyuk and R. M. Narayanan, "ECCM capabilities of an ultrawideband bandlimited random noise imaging radar," IEEE Transactions on Aerospace and Electronic Systems, vol. 38, no. 4, pp. 1243-1255, 2002.

[6] S. L. Johnston, "Radar electronic counter-countermeasures," IEEE Transactions on Aerospace and Electronic Systems, vol. 14, no. 1, pp. 109-117, 1978.

[7] J.-M. Guo, J.-X. Li, and Q. Lv, "Survey on radar ECCM methods and trends in its developments," in Proceedings of CIE International Conference of Radar (ICR '06), pp. 1-4, Shanghai, China, October 2006.

[8] M. P. Grant, G. R. Cooper, and A. K. Kamal, "A class of noise radar systems," Proceedings of the IEEE, vol. 51, pp. 1060-1061, 1963.

[9] L. Guosui, G. U. Hong, and S. U. Weimin, “The development of random signal radars," IEEE Transactions on Aerospace and Electronic Systems, vol. 35, no. 3, pp. 770-777, 1999.

[10] K. Kulpa, K. Lukin, W. Miceli, and T. Thayaparan, "Signal processing in noise radar technology," IET Radar, Sonar and Navigation, vol. 2, no. 4, pp. 229-232, 2008.
[11] D. S. Garmatyuk and R. M. Narayanan, "Ultra-wideband continuous-wave random noise Arc-SAR," IEEE Transactions on Geoscience and Remote Sensing, vol. 40, no. 12, pp. 25432552, 2002.

[12] M. Speth, S. A. Fechtel, G. Fock, and H. Meyr, "Optimum receiver design for wireless broad-band systems using OFDMpart I," IEEE Transactions on Communications, vol. 47, no. 11, pp. 1668-1677, 1999.

[13] J. C.-I. Chuang and N. Sollenberger, "Beyond 3G: wideband wireless data access based on OFDM and dynamic packet assignment," IEEE Communications Magazine, vol. 38, no. 7, pp. 78-87, 2000.

[14] A. Batra, J. Balakrishnan, G. R. Aiello, J. R. Foerster, and A. Dabak, "Design of a multiband OFDM system for realistic UWB channel environments," IEEE Transactions on Microwave Theory and Techniques, vol. 52, no. 9 I, pp. 2123-2138, 2004.

[15] N. Levanon, "Multifrequency complementary phase-coded radar signal," IEE Proceedings: Radar, Sonar and Navigation, vol. 147, no. 6, pp. 276-284, 2000.

[16] E. Mozeson and N. Levanon, "Multicarrier radar signals with low peak-to-mean envelope power ratio," IEE Proceedings: Radar, Sonar and Navigation, vol. 150, no. 2, pp. 71-77, 2003.

[17] D. S. Garmatyuk, "Simulated imaging performance of UWB SAR based on OFDM," in Proceedings of IEEE International Conference on Ultra-Wideband (ICUWB '06), pp. 237-242, Waltham, Mass, USA, September 2006.

[18] G. E.A. Franken, H. Nikookar, and P. Van Genderen, "Doppler tolerance of OFDM-coded radar signals," in Proceedings of the 3rd European Radar Conference (EuRAD '06), pp. 108-111, Manchester, UK, September 2006.

[19] M. Ruggiano and P. Van Genderen, "Wideband ambiguity function and optimized coded radar signals," in Proceedings of the 4th European Radar Conference (EURAD '07), pp. 142-145, Munich, Germany, October 2007.

[20] M. A. Sebt, A. Sheikhi, and M. M. Nayebi, "Orthogonal frequency-division multiplexing radar signal design with optimised ambiguity function and low peak-to-average power ratio," IET Radar, Sonar and Navigation, vol. 3, no. 2, pp. 122132, 2009.

[21] D. Garmatyuk, J. Schuerger, K. Kauffman, and S. Spalding, "Wideband OFDM system for radar and communications," in Proceedings of IEEE National Radar Conference, Pasadena, Calif, USA, May 2009.

[22] M. Roberten and E. R. Brown, "Integrated radar and communications based on chirped spread-spectrum techniques," in Proceedings of IEEE MTT-S International Microwave Symposium Digest, vol. 1, pp. 611-614, Philadelphia, Pa, USA, June 2003.

[23] N. Levanon and E. Mozeson, Radar Signals, John Wiley \& Sons, Hoboken, NJ, USA, 2004.

[24] S. V. Maric and E. L. Titlebaum, "A class of frequency hop codes with nearly ideal characteristics for use in multipleaccess spread-spectrum communications and radar and sonar systems," IEEE Transactions on Communications, vol. 40, no. 9, pp. 1442-1447, 1992.

[25] S. Zoican, "Frequency hopping spread spectrum technique for wireless communication systems," in Proceedings of the 5th IEEE International Symposium on Spread Spectrum Techniques \& Applications (IEEE ISSSTA '98), vol. 1, pp. 338-341, Sun City, South Africa, September 1998.

[26] S. Vitebskiy, L. Carin, M. A. Ressler, and F. H. Le, "Ultrawideband, short-pulse ground-penetrating radar: simulation and measurement," IEEE Transactions on Geoscience and Remote Sensing, vol. 35, no. 3, pp. 762-772, 1997. 
[27] D. C. Lush and D. A. Hudson, "Ambiguity function analysis of wideband radars," in Proceedings of the IEEE National Radar Conference, pp. 16-20, Los Angeles, Calif, USA, March 1991.

[28] G. N. Saddik, R. S. Singh, and E. R. Brown, "Ultra-wideband multifunctional communications/radar system," IEEE Transactions on Microwave Theory and Techniques, vol. 55, no. 7, pp. 1431-1436, 2007.

[29] P. M. Oliveira and V. Barroso, "On the concept of instantaneous frequency," in Proceedings of IEEE International Conference on Acoustics, Speech and Signal Processing (ICASSP '98), vol. 4, pp. 2241-2244, Seattler, Wash, USA, May 1998.

[30] G. Girolami and D. Vakman, "Instantaneous frequency estimation and measurement: a quasi-local method," Measurement Science and Technology, vol. 13, no. 6, pp. 909-917, 2002.

[31] J. Schuerger and D. Garmatyuk, "Deception jamming modeling in radar sensor networks," in Proceedings of IEEE Military Communications Conference (MILCOM '08), Washington, DC, USA, November 2008.

[32] M. Soumekh, Synthetic Aperture Radar Signal Processing, John Wiley \& Sons, New York, NY, USA, 1999. 